\section{The Meaning in Quality of Life}

\author{
Santosh K. Chaturvedi ${ }^{1} \cdot$ Krishna Prasad Muliyala $^{1}$
}

Published online: 10 November 2016

(C) Springer India Pvt. Ltd. 2016

This current issue has been envisaged to be a special issue on Quality of life (QOL) in mental health. It has been successful to a certain extent, with a thought provoking Guest editorial by a well-known expert in this area, Professor A. George Awad. Incidentally, this issue also includes a book review on a book edited by Professor Awad and Lakshmi Voruganti, entitled, Beyond Assessment of Quality of Life in Schizophrenia, a very useful collection, indeed, published by Springers International Publishing. The Guest editorial is followed by a review article by Professor Mary V. Seeman on Hierarchy and quality of life among psychiatric users. Other interesting articles on quality of life include a Comparative study of quality of life, social support and dysfunction in alcohol dependent men attending a de-addiction clinic in India, and Wellness as a means for better quality of life in schizophrenia. The issue also has contributions from Canada from north America and Brazil in south America on Addressing workplace accommodations for people with mental illness, University of Toronto, Canada and the Brazilian community mental health care services: social inclusion and psychosocial rehabilitation, Sao Paulo, Brazil. There are also two articles for the United Kingdom, one, Useful groups like this don't usually happen in places like these: an evaluation of psychoeducational and skills workshops within inpatient rehabilitation services, and another Mixed staff and client mindfulness groups in a long stay inpatient setting: an evaluation. There is also a study on patient satisfaction and another on caregiver burden, in this issue. It is a good

Santosh K. Chaturvedi

skchatur@gmail.com

1 Psychiatric Rehabilitation Services, National Institute of Mental Health and Neurosciences, Bengaluru, India collection of significant contributions towards quality of life as well as mental health rehabilitation.

Till date, in medical practice, objective clinical examination and investigations are used as indicators of the outcome of a disease and efficacy of therapeutic interventions. Though these are integral component of modern medicine, importance of patient's point of view and social perspective in the therapeutic process cannot be ignored. Quality of life in medicine assesses such perspectives. With enhanced life expectancy, the medical focus has shifted from merely prolonging life to improving quality of life. Over the last few decades, the concept quality of life has given complete image makeover to clinical medicine including mental health. There has been tremendous growth in the number of research papers on quality of life in chronic mental illnesses and it has given much needed voice to the patient's perspective in the face of the traditional paternalistic health care system.

As a broad, general concept, quality of life has included several domains related to health, but the concept also originally included many other non-health-related issues such as work, family, prosperity, spirituality, and environment. Quality of life in mental health began to gain momentum with increasing concerns about the unsatisfactory life conditions of patients suffering from chronic mental illnesses in the community. Greater emphasis is given on quality of life as an outcome measure for comparison of therapies and programmes as well as for equitable distribution of resources. The recent interest in QOL has been generated due to two main reasons, firstly as a part of the larger drive towards "Health for all" and the promotion of physical, mental and social well-being, and secondly, a fundamental rethinking of the goal of rehabilitation as an indicator of good QOL, as perceived by the patients and their relatives. 
Historically, the concept of QOL has its beginnings with cancer treatments. The severe toxicity related to anticancer chemotherapy, mutilating surgery and adverse effects of radiation therapy to prolong life and increase survival, made the patients, their caregivers and the health professionals wonder the choice between 'quantity of life' versus 'quality of life'. The price of longer survival was paid through poor quality of life. Ever since, the search is on for effective and less toxic cancer treatment methods, drugs, and surgical procedures.

Similarly, in the field of mental health, in the 1960's, as a consequence of the shift towards deinstitutionalization, it was believed that the psychiatric patient's QOL would be greatly enhanced by living with the family. Later, the concept of burden of the family members or caregivers emerged. Burden was measured in both subjective and objective domains. Several factors such as the type of symptoms, patients' physical or social disability, social support, social relationships and coping mechanisms were shown to be related to burden perceived by the caregivers. Burden also had some relation with quality of life.

There is no consensus regarding what is quality of life. Similarities between satisfaction and quality of life are apparent. Nevertheless they are not the same. Quality of life and standard of living are also related concepts. Lastly, in many societies, quality of life is considered at par with the functional status. Quality of life is not a unitary concept. QOL is a complex, multifaceted concept which continues to defy consensual definition and has multiple interpretations.

Current concepts range from those with a holistic emphasis on the social, environmental, and physical wellbeing of patients after treatment to those that describe the impact of a person's health on his/her ability to lead a fulfilling life. There are widely valued aspects of life that are not generally regarded as health, including income, freedom, and social support. Although low income, lack of freedom and poor social support may be relevant to health, these are excluded when dealing with quality of life and health problems and there is focus on disease related aspects of functional capacity (e.g. mobility in pulmonary disease) and well-being. For this purpose, the term 'healthrelated quality of life (HRQOL)' was coined. Additionally, many investigators seem to substitute QOL for other terms intended either to describe patients' health (such as 'health/functional status') or to summarize those aspects of life quality or function which are impacted by one's health status (the 'health-related QOL').

Thus, QOL is a much broader concept which consists of both medical and psychosocial aspects, including activities of daily living, instrumental activities, psychological wellbeing, social functioning, and perception of health status, pain, and overall satisfaction with life. In the end, it seems,
QOL is something which everyone understands what it is, but finds it difficult to describe or define accurately!

Several studies have shown that there is often disagreement between physicians and patients on the severity of symptoms and on the success of medical treatment. Clinicians generally base their assessment of the results of their treatment on the basis of symptomatic improvement. In contrast, patients are more likely to evaluate the outcome of treatment according to their personal sense of well being. By definition, quality of life is a subjective construct comprising of self reporting by patients and their subjective judgment which requires a degree of cognitive ability. However, subjective reports by patients suffering from chronic mental illnesses are largely ignored by the clinicians because of their impaired judgment, insight and neurocognitive deficits. Several research publications have supported the idea that subjective self-reports can be both measured and reliably quantified.

In the field of mental health, quality of life assessment must be carried out not only by the patient but also by professional helpers and key informants, as a rule family members and friends of the patients. The emphasis of quality of life varies from individual's feelings of wellbeing to physical, social, occupational, spiritual, marital and sexual functioning. It is generally agreed that in the final analysis the determination of a person's quality of life is a matter for the individual to decide. The quality of life is so personal and subjective that another person's assessment may be deceptive. Whose life is it, anyway?

Among other closely related concepts, functional status refers to entire domain of functioning to provide for the necessities of life. Although the domains of functional status are similar to QOL, functional status doesn't account for subjective interpretation and is measured only objectively. Conversely, life satisfaction is a purely subjective measure. Satisfaction with life fails to consider objective indicators which are critical attributes of QOL. Well-being and QOL cannot be synonymous because wellbeing is purely subjective and QOL has both subjective and objective attributes.

The time of assessment of quality of life is also important. In cancer, it should be done at the time of diagnosis and at multiple points during diagnosis, investigations, pre- and post-treatment, and later during follow-up irrespective of whether the treatment has been successful or not. In advanced disease, QOL assessment needs to be done regularly to check that the palliative measures make life as comfortable for the patient as possible. Likewise in persons with chronic mental health problems, QOL needs to be assessed at multiple intervals. Many psychiatric medications adversely affect QOL, and some improve QOL, by reducing the adverse drug side effects! The concept of 'quality of life' extends beyond a balance 
between the impact of a treatment and its side-effects, to recognize and respect the autonomous individual-the patient-in body, mind and spirit' in the context of social relationships with family and friends. When we assess the usefulness of any therapeutic intervention in advanced diseases, it is not enough to go by the physical factors. The patient's subjective feelings, hopes and fears, must be taken into consideration.

In developing countries, besides the distressing symptoms of the illness, a majority of patients experience financial, domestic, social and occupational difficulties. It also must be acknowledged that often the cost of treatment could possibly have a greater negative effect on the quality of subsequent life than any positive effect of the offered treatment. A way of routine clinical assessment of QOL in day to day clinical practice would improve patient satisfaction. It is observed that QOL assessment improves quality of life, somehow. Meaning seems to have more value in quality of life.

A day long Symposium and national conference was held earlier this year at Bangalore, and the proceedings were also printed. Future issues of the journal would share the presentations and proceedings, to keep alive the discussion on quality of life in chronic mental illness. 